\title{
Fortalecimiento de las alianzas docencia-servicio para el avance de la enfermería en Colombia*
}

\section{Strengthening of Teaching-service Partnerships for the Progress of Nursing in Colombia}

\section{Fortalecimento de parcerias ensino-serviço para o avanço da enfermagem na Colômbia}

Fecha de recepción: 08-07-2016 Fecha de aceptación: 09-03-2017 Disponible en línea: 30-05-2017 doi:10.11144/Javeriana.rgps16-32.fads

Cómo citar este artículo:

Carvajal-Hermida EY, Pérez-Giraldo B, Sánchez-Herrera B. Fortalecimiento de las alianzas docencia-servicio para el avance de la enfermería en Colombia. Rev Gerenc Polít Salud. 2017; 16 (32): 96-107. https:// doi.org/10.11144/Javeriana.rgps16-32.fads

\author{
Elsa Yolanda Carvajal-Hermida** \\ Beatriz Pérez-Giraldo*** \\ Beatriz Sánchez-Herrera****
}

\footnotetext{
* Artículo de revisión.

** Enfermera de la Cruz Roja Colombiana, magíster en Enfermería Pediátrica de la Pontificia Universidad Javeriana, directora de Enfermería del Hospital Universitario Fundación Santa Fe de Bogotá. Correo electrónico: elsayolandacarvajal@gmail.com

*** Enfermera de la Pontificia Universidad Javeriana, magíster en Enfermería de la Universidad Nacional de Colombia, directora de Carrera de la Facultad de Enfermería y Rehabilitación de la Universidad de la Sabana. Correo electrónico: beatriz.perez@unisabana.edu.co

**** Enfermera de la Pontificia Universidad Javeriana, Master in Science of Nursing, University of Pennsylvania, profesora de alto prestigio académico de la Facultad de Enfermería y Rehabilitación de la Universidad de la Sabana. Profesora titular (p) y especial de la Universidad Nacional de Colombia. Correspondencia: Beatriz Sánchez-Herrera, Facultad de Enfermería y Rehabilitación, Universidad de La Sabana, Chía, Cundinamarca, Colombia. Correo de correspondencia: clara.sanchez@unisabana.edu.co
} 


\section{Resumen}

Objetivo: revisar las tendencias conceptuales, teóricas y empíricas sobre el fortalecimiento de alianzas docencia-servicio para el avance de la enfermería en Colombia. Método: revisión sistemática de literatura. Resultados: el rastreo bibliográfico entre los años 2013 y 2015, bajo los descriptores enfermería, práctica avanzada y educación o docencia, dio como resultado veintitrés artículos analizados en su totalidad. Conclusiones: hay predominio de abordajes conceptuales y teóricos para el análisis de la práctica de enfermería. A efectos de fortalecer la política de alianza docencia-servicio se recomienda trabajar por competencias, mejorar la relación con el paciente y mirar integralmente el sistema. La mayor formación de enfermería en la clínica, la cualifica y le exige nuevos retos de autonomía. La alianza docencia-servicio es necesaria para la cualificación de la práctica de enfermería en Colombia.

Palabras clave: enfermería; enfermería de práctica avanzada; educación en enfermería; política de educación superior; política de salud; convenios

\section{Abstract}

Objective: To review the conceptual, theoretical and empirical trends on the strengthening Teaching-Service Partnerships for the progress of nursing in Colombia. Method: Systematic review of literature. Results: The bibliographic tracking was performed between 2013 and 2015, under the descriptors Nursing, Advanced Practice and Education or Teaching. The search resulted in 23 articles, which were fully analyzed. Conclusions: There is a predominance of conceptual and theoretical analysis of nursing practice approaches. In order to strengthen the Policy of Teaching-Service Partnerships it is recommended to work by competencies, to improve the relationship with the patient, and to approach the Health system fully. To have the highest nursing education in the clinic, means to qualify nursing and to approach new challenges and demands of autonomy. Strengthening Teaching-Service Partnerships is necessary for the qualification of nursing practice in Colombia.

Keywords: nursing; advanced practice nursing; education, nursing; higher education policy, health policy; joint ventures

\section{Resumo}

Objetivo: revisar as tendências conceituais, teóricas e empíricas sobre o fortalecimento de parcerias ensino-serviço para o avanço da enfermagem na Colômbia. Método: revi sao sistemática de literatura. Resultados: rasteio bibliográfico entre 2013 e 2015, sob os descritores enfermagem, prática avançada e ensino ou docência, deu como resultado vinte e três artigos analisados na sua totalidade. Conclusões: tem predomínio de abordagens conceituais e teóricas para análise da prática de enfermagem. A fim de reforçar a política de parcerias ensino-serviço recomenda-se trabalhar por competências, melhorar o relacionamento com o paciente e olhar de maneira abrangente o sistema. A maior formação de enfermagem na clínica, a qualifica e exige novos desafios de autonomia. A parceria ensino-serviço é precisa para a qualificação da prática de enfermagem na Colômbia.

Palavras-chave: enfermagem; enfermagem de prática avançada; ensino em enfermagem; política de ensino superior; política de saúde; convênios 


\section{Introducción}

La práctica de enfermería como punto de encuentro entre sus componentes disciplinares y profesionales es sin duda el escenario más relevante (1). En ella se deben reflejar los avances teóricos y es allí donde surgen los problemas que la enfermería debe investigar para cualificar su acción.

Aunque el rol asistencial es sin duda fundamental para la práctica, de manera errónea en ocasiones se denomina práctica exclusivamente al quehacer de la enfermera en la asistencia. Vale la pena señalar que la práctica de enfermería ha sido descrita mediante el ejercicio de diez roles que incluyen el asistencial o de cuidador, el de educador, el de consultor, el de investigador, el de innovador o agente de cambio interno, el político o agente de cambio externo, el de abogado, el administrativo o gestor del cuidado, el de colaborador o rol interdisciplinario y el de líder, que a pesar de ser transversal como muchos otros, con frecuencia es un rol en sí mismo (2). Esta multiplicidad de roles se reconoce incluso dentro de la práctica clínica de enfermería (3).

De otra parte, la convergencia entre los avances teóricos y la práctica de la enfermería ha sido planteada como necesaria durante muchos años. En Colombia, los inicios de la profesionalización de enfermería se vinculan a los avances teóricos que le dieron identidad (4). El camino de la profesionalización dio paso a la búsqueda del profesionalismo donde se hace cada vez más evidente la necesidad de cualificar la práctica del cuidado (5). Si bien por mucho tiempo se pensó que el rol protagónico debería ser asumido por la academia (6), la enfermería ha venido comprendiendo que para cualificar la práctica es preciso fortalecer la política de trabajo conjunto entre los escenarios docentes y los asistenciales.
De manera simultánea, la disciplina profesional de enfermería ha sido influida por un proceso general de globalización caracterizado por señales de transculturización, empleo de modernas tecnologías de información y comunicación (TIC), mayor acceso al conocimiento y en algunos casos a redes y recursos internacionales. En medio de este cambio, se ha buscado la esencia profesional centrada en el cuidado (7). A pesar de ello, el análisis de la enfermería como profesión reclama mayor conocimiento, definición de metodologías propias, incremento del nivel educativo, fortalecimiento de la autonomía y de la ética y, sin duda, una contribución social propia y visible (8).

En tal sentido, un análisis de las tendencias de cualificación de la práctica de enfermería, mediante una revisión sistemática (9), puede ser útil para fortalecer la política vigente de docencia-servicio. De una parte, esta política ofrece un sinnúmero de posibilidades al poner la teoría y la práctica en contacto (10); de otra, permite enfrentar los riesgos propios de los sistemas de salud cuyo enfoque sea diferente al de servicio (11).

A escala local se encuentran algunas evidencias que señalan la relevancia del vínculo docencia-servicio (12). En cuanto a la normativa, tanto el decreto presidencial que regula dicha relación para los programas de formación de talento humano del área de la salud en Colombia, Decreto 2376 de 2010 (13), como la Resolución del Ministerio de Salud y Protección Social 3409 de 2012, que define la documentación a efectos del reconocimiento de las instituciones prestadoras de servicios de salud como hospital universitario (14), han demostrado ser orientadoras en el campo.

Las asociaciones y agrupaciones científicas y gremiales de enfermería en Colombia se han ocupado parcialmente del tema. La 
Asociación Colombiana de Facultades y Escuelas de Enfermería (Acofaen) señaló que es evidente una desarticulación en la relación docencia-servicio, lo cual conlleva una falta de correspondencia entre la formación y las necesidades de la asistencia en la cual no se hace evidente la existencia de nuevos modelos pedagógicos y el avance conceptual de la enfermería. A partir de ello, el plan de desarrollo vigente (2009-2018) propone generar lineamientos para propiciar una relación docencia-servicio que respalde mejor el desarrollo de la enfermería, a partir de las normas actuales, con respecto a las cuales considera importante el acompañamiento a "las unidades académicas" (15-16).

No se conocen planteamientos sobre el particular de otras instancias de carácter nacional como la Asociación Nacional de Enfermeras de Colombia (ANEC), el Tribunal de Ética en Enfermería, el Capítulo Colombiano de la Sociedad de Honor de Enfermería Sigma Theta Tau, o los grupos de trabajo conjunto entre instituciones de carácter asistencial (17).

En los registros del Departamento Administrativo de Ciencia, Tecnología e Innovación (Colciencias) son evidentes algunos esfuerzos por el avance y el desarrollo de la disciplina profesional de enfermería. Algunos casos podrían reflejar cercanía o generar la oportunidad de trabajo conjunto entre la docencia y la asistencia, e incluyen a la fecha 41 grupos (véase tabla 1). Sin embargo, y a pesar de tener evidencia de trabajos conjuntos (18), llama la atención que ninguno de los grupos sea registrado desde una institución asistencial.

Tabla 1. Grupos de inVestigación Registrados en Colciencias relacionados con EL DESARROLLO DE LA ENFERMERÍA EN COLOMBIA

\begin{tabular}{|c|c|c|}
\hline Grupo & Institución & Líder \\
\hline Cuidado de Enfermería & Universidad de La Sabana & Beatriz Pérez Giraldo \\
\hline Cuidado de Enfermería & Universidad del Valle & Martha Lucía Vásquez Truissi \\
\hline $\begin{array}{l}\text { Conceptualización y } \\
\text { Práctica de Enfermería }\end{array}$ & Pontificia Universidad Javeriana & Fabiola Castellanos Soriano \\
\hline $\begin{array}{l}\text { Grupo de investigación } \\
\text { la práctica de enfermería } \\
\text { en el contexto social }\end{array}$ & Universidad de Antioquia & María Victoria Arteaga Henao \\
\hline $\begin{array}{l}\text { Grupo de investigación } \\
\text { de enfermería Everest }\end{array}$ & Universidad de Santander & Myriam Durán Parra \\
\hline $\begin{array}{l}\text { Grupo de investigación } \\
\text { de Enfermería }\end{array}$ & Universidad del Norte & Gloria Cecilia Visbal Illera \\
\hline $\begin{array}{l}\text { Grupo de investigación } \\
\text { Exclusiones y } \\
\text { Resistencias en el } \\
\text { Cuidado de la Salud }\end{array}$ & $\begin{array}{l}\text { Universidad Pedagógica y } \\
\text { Tecnológica de Colombia }\end{array}$ & No especificado \\
\hline $\begin{array}{l}\text { Grupo de Investigación } \\
\text { VisageI }\end{array}$ & Universidad Mariana & No especificado \\
\hline $\begin{array}{l}\text { Grupo de Investigación } \\
\text { en Enfermería }\end{array}$ & Universidad Católica de Manizales & Claudia Liliana Valencia Rico \\
\hline $\begin{array}{l}\text { Perspectivas del } \\
\text { Cuidado de Enfermería }\end{array}$ & $\begin{array}{l}\text { Fundación Universitaria de } \\
\text { Ciencias de la Salud }\end{array}$ & Ana Julia Carrillo Algarra \\
\hline
\end{tabular}




\begin{tabular}{|c|c|c|}
\hline Grupo & Institución & Líder \\
\hline $\begin{array}{l}\text { Grupo de Investigadores } \\
\text { de Enfermería de } \\
\text { Risaralda }\end{array}$ & $\begin{array}{c}\text { Fundación Universitaria del Área } \\
\text { Andina }\end{array}$ & Nelcy Muñoz Astudillo \\
\hline $\begin{array}{l}\text { Salud sexual y } \\
\text { Reproductiva } \\
\text { Enfermería Unbosque }\end{array}$ & Universidad El Bosque & $\begin{array}{l}\text { Miguel Antonio Sánchez } \\
\text { Cárdenas }\end{array}$ \\
\hline $\begin{array}{l}\text { Ciencias del Cuidado en } \\
\text { Enfermería }\end{array}$ & Universidad del Magdalena & Ediltrudis Ramos de la Cruz \\
\hline $\begin{array}{l}\text { Grupo de Investigación } \\
\text { en Enfermería } \\
\text { Comunidad-Salud y } \\
\text { Educación }\end{array}$ & $\begin{array}{l}\text { Universidad Cooperativa de } \\
\text { Colombia }\end{array}$ & Gisela Esther González Ruiz \\
\hline $\begin{array}{l}\text { Grupo de investigación } \\
\text { en enfermería: } \\
\text { investigación en } \\
\text { salud, innovación } \\
\text { biotecnológica, } \\
\text { educación y cultura } \\
\text { (Gisibec) }\end{array}$ & $\begin{array}{c}\text { Corporación Universitaria Rafael } \\
\text { Núñez }\end{array}$ & Anderson Díaz Pérez \\
\hline $\begin{array}{l}\text { Educación en } \\
\text { Enfermería Grieeq }\end{array}$ & Universidad del Quindío & Carmen Aydé Fernández Rincón \\
\hline $\begin{array}{l}\text { Cuidado y práctica } \\
\text { en enfermería, salud } \\
\text { familiar enfermería } \\
\text { familiar y medición en } \\
\text { salud }\end{array}$ & Universidad Nacional de Colombia & Virginia Inés Soto Lesmes \\
\hline Cuidado de la Salud & Universidad Santiago de Cali & Estela Rodríguez Torres \\
\hline $\begin{array}{l}\text { Grupo de Investigación } \\
\text { en Dolor y Cuidado } \\
\text { Paliativo }\end{array}$ & $\begin{array}{l}\text { Universidad Pontificia } \\
\text { Bolivariana-Sede Medellín }\end{array}$ & Alicia Krikorian Daveloza \\
\hline $\begin{array}{l}\text { Cuidado de Enfermería } \\
\text { al Paciente Crónico }\end{array}$ & Universidad Nacional de Colombia & Olivia Lorena Chaparro Díaz \\
\hline $\begin{array}{l}\text { Cuidado Cultural de la } \\
\text { Salud }\end{array}$ & Universidad Nacional de Colombia & Alba Lucero López Díaz \\
\hline $\begin{array}{l}\text { Cuidado } \\
\text { Cardiorrespiratorio }\end{array}$ & Universidad Manuela Beltrán & Ana Isabel García Muñoz \\
\hline $\begin{array}{l}\text { Grupo de Investigación } \\
\text { en Cuidado }\end{array}$ & $\begin{array}{c}\text { Universidad Pontificia } \\
\text { Bolivariana-Sede Medellín }\end{array}$ & Dora Isabel Giraldo Montoya \\
\hline Cuidado en Salud & $\begin{array}{l}\text { Universidad Libre de } \\
\text { Colombia-Cali }\end{array}$ & María Oliva Sánchez Ortiz \\
\hline $\begin{array}{l}\text { Cuidado a la Salud de } \\
\text { los Colectivos }\end{array}$ & Universidad de Cartagena- & Zuleima Cogollo Milanés \\
\hline $\begin{array}{l}\text { Cuidado Peri } \\
\text { Operatorio }\end{array}$ & Universidad Nacional de Colombia & Rosibel Prieto Silva \\
\hline $\begin{array}{l}\text { Urgencias y Cuidado } \\
\text { Crítico }\end{array}$ & Universidad Nacional de Colombia & Sandra Rocío Guáqueta Parada \\
\hline Gerencia del Cuidado & $\begin{array}{l}\text { Universidad Libre de } \\
\text { Colombia-Pereira }\end{array}$ & Lilia Andrea Buitrago Malaver \\
\hline
\end{tabular}




\begin{tabular}{|c|c|c|}
\hline Grupo & Institución & Líder \\
\hline Cuidado a las Personas & Universidad de Cartagena & Amparo Montalvo Prieto \\
\hline $\begin{array}{l}\text { Cuidado Materno } \\
\text { Perinatal }\end{array}$ & Universidad Nacional de Colombia & Myriam Patricia Pardo Torres \\
\hline $\begin{array}{l}\text { Cuidado para la Salud } \\
\text { Cardiorrespiratoria }\end{array}$ & Universidad Nacional de Colombia & Magda Lucía Flórez \\
\hline $\begin{array}{l}\text { Salud y cuidado de } \\
\text { los colectivos }\end{array}$ & Universidad Nacional de Colombia & Alba Idaly Muñoz Sánchez \\
\hline $\begin{array}{l}\text { Cuidado de La } \\
\text { Salud-UT }\end{array}$ & Universidad del Tolima & $\begin{array}{l}\text { Madeleine Cecilia Olivella } \\
\text { Fernández }\end{array}$ \\
\hline Cuidado de Enfermería & Universidad Simón Bolívar & Gloria Elena Lastre Amell \\
\hline $\begin{array}{l}\text { Cuidado de la Salud y } \\
\text { Desarrollo Sostenible }\end{array}$ & $\begin{array}{c}\text { Universidad de Ciencias Aplicadas } \\
\text { y Ambientales }\end{array}$ & Nelly Cáliz Romero \\
\hline $\begin{array}{l}\text { Cuidado Integral de la } \\
\text { Salud Humana }\end{array}$ & Universidad Manuela Beltrán & Luz Evelsy Ladino Gil \\
\hline $\begin{array}{l}\text { Cuidado de la Salud en } \\
\text { el Contexto Individual, } \\
\text { Familiar y Social }\end{array}$ & Fundación Universitaria Sanitas & Astrid Viviana Robayo Téllez \\
\hline Cuidado de La Salud & Universidad de Sucre & Luz Marina García \\
\hline $\begin{array}{l}\text { Grupo de Estudios e } \\
\text { Investigación para el } \\
\text { Cuidado del Niño con } \\
\text { Heridas, Estomas e } \\
\text { Incontinencias }\end{array}$ & Universidad Nacional de Colombia & Nidia Sandra Guerrero Gamboa \\
\hline $\begin{array}{l}\text { Salud, Cuidado y } \\
\text { Sociedad }\end{array}$ & Unidad Central del Valle del Cauca & Luz Damaris Rojas Rodríguez \\
\hline Cuidado y Vida & Universidad de Cartagena & Estela Melguizo Herrera \\
\hline
\end{tabular}

Fuente: elaboración propia con base en información registrada en Colciencias (19)

Con relación al insuficiente desarrollo del trabajo nacional con respecto al fortalecimiento de las alianzas docencia-servicio, a pesar de las insistentes recomendaciones para acercar la teoría a la práctica como la mejor forma de cualificar la enfermería (2), el presente trabajo, realizado por la Dirección de Enfermería de la Fundación Santa Fe de Bogotá y la Facultad de Enfermería y Rehabilitación de la Universidad de la Sabana, en el marco de una iniciativa liderada por el Grupo gestor para el fortalecimiento de la alianza docencia servicio para el desarrollo de la enfermería en Colombia, buscó mirar las tendencias en el ámbito internacional para generar, a partir de ello, un referente que tener en cuenta en el fortalecimiento de esta política.

\section{Metodología}

Para desarrollar el presente trabajo se realizó una búsqueda sistemática siguiendo los planteamientos de Beltrán (9), quien establece cinco pasos:

1. Definir la pregunta orientadora de la búsqueda y su objetivo. La pregunta orientadora de la búsqueda: ¿Cuáles son las tendencias conceptuales, teóricas y empíricas que reporta la literatura en el ámbito 
internacional en relación con el fortalecimiento de alianzas docencia-servicio para el avance de la enfermería? Con ello se pretendió lograr el objetivo de revisar las tendencias conceptuales, teóricas y empíricas en relación con el fortalecimiento de alianzas docencia-servicio para el avance de la enfermería en Colombia.
2. Identificación de la información y localización de los estudios. La búsqueda de los estudios se realizó definiendo la ventana de observación bajo criterios establecidos para los descriptores, bases de datos consultadas, tipo, tiempo e idioma de las publicaciones y tipo de estudio (véase tabla 2).

Tabla 2. Criterios de la búsqueda de tendencias en el fortalecimiento de alianzas DOCENCIA-SERVICIO PARA EL AVANCE DE LA ENFERMERÍA

\begin{tabular}{|c|c|}
\hline Criterio & Definición para la búsqueda \\
\hline \multirow{6}{*}{$\begin{array}{l}\text { Descriptor } \\
\text { DeCS }\end{array}$} & $\begin{array}{l}\text { Enfermería: campo de atención de enfermería referido a la promoción, el manteni- } \\
\text { miento y la restauración de la salud. }\end{array}$ \\
\hline & $\begin{array}{l}\text { Educación: adquisición de conocimientos como resultado de la instrucción en un } \\
\text { curso formal de estudios. Usado para educación, programas de entrenamiento, } \\
\text { cursos de varios campos y disciplinas y capacitación de grupos de personas. } \\
\text { Enseñanza: proceso educacional de instrucción. }\end{array}$ \\
\hline & $\begin{array}{l}\text { Práctica: realización de un acto una o más veces, con vistas a su fijación o mejora; } \\
\text { cualquier realización de un acto o comportamiento que lleva al aprendizaje. }\end{array}$ \\
\hline & $\begin{array}{l}\text { Al hablar de práctica profesional se hace referencia a la utilización de los conoci- } \\
\text { mientos propios de una profesión particular, en tanto que la práctica de la medici- } \\
\text { na es el ejercicio de los conocimientos propios para la identificación y el tratamiento } \\
\text { práctico de las enfermedades. Incluye, en el caso del campo de la biomedicina, a las } \\
\text { actividades profesionales relacionadas con la atención de salud y el desempeño pro- } \\
\text { piamente dicho de los deberes relacionados con la provisión de la atención de salud. }\end{array}$ \\
\hline & $\begin{array}{l}\text { Al hablar de enfermería, la práctica hace referencia a la práctica de la enferme- } \\
\text { ría por personas licenciadas, no registradas, calificadas para proporcionar aten- } \\
\text { ción de rutina a enfermos. }\end{array}$ \\
\hline & $\begin{array}{l}\text { Avanzada: la práctica avanzada de enfermería significa que esta es una atención sa- } \\
\text { nitaria basada en la evidencia, fundada en la investigación y la erudición. Los } \\
\text { profesionales incluyen profesionales en enfermería, especialistas en enfermería } \\
\text { clínica, enfermeras anestesistas y matronas. }\end{array}$ \\
\hline $\begin{array}{l}\text { Bases de datos } \\
\text { consultadas } \\
\text { para hacer la } \\
\text { búsqueda }\end{array}$ & $\begin{array}{l}\text { El acceso a las siguientes quince bases de datos se hizo mediante el metabuscador } \\
\text { Descubridor, del Sistema Nacional de Bibliotecas de la Universidad Nacional de } \\
\text { Colombia. Se incluyeron: Scielo, Medline With Full Text, Sciencedirect, Infotrac } \\
\text { Health Reference Center Academic, Academic Onefile, Business Source Complete, } \\
\text { British Library Ethos, Expanded Academic Asap, Health \& Wellness Resource } \\
\text { Center, Psycarticles, General Onefile, Informit Health Collection, Econlit With Full } \\
\text { Text, Library, Information Science \& Technology Abstracts, Science In Context. }\end{array}$ \\
\hline $\begin{array}{l}\text { Tipo de } \\
\text { publicaciónes }\end{array}$ & Se selecionaron solamente publicaciones de tipo académico que fueran arbitradas. \\
\hline Tiempo & $\begin{array}{l}\text { Enero 2013-diciembre de 2015. Se tomó un periodo de tres años, buscando con ello do- } \\
\text { cumentar el tema de manera suficiente, empleando experiencias recientes en el campo. }\end{array}$ \\
\hline Tipo de estudio & $\begin{array}{l}\text { Por la naturaleza del fenómeno en estudio, que considera planteamientos de tipo } \\
\text { conceptual, teórico, se dejó abierto a publicación de investigación cualitativa, cuan- } \\
\text { titativa o mixta, análisis, revisión o reflexión. }\end{array}$ \\
\hline Idiomas & Inglés y español \\
\hline
\end{tabular}

Fuente: elaboración propia con base en el Directorio de Términos de la Salud DeCS, 2015 
3. Selección de artículos. A partir de parámetros establecidos se desarrollaron las siguientes fórmulas de búsqueda para poder precisar el fenómeno en estudio (véase tabla 3).

Tabla 3. Fórmulas de búsqueda de tendencias en El Fortalecimiento DE ALIANZAS DOCENCIA-SERVICIO PARA EL AVANCE DE LA ENFERMERÍA

\begin{tabular}{|c|c|}
\hline Fórmulas de búsqueda & $\begin{array}{l}\text { Artículos } \\
\text { reportados }\end{array}$ \\
\hline $\begin{array}{l}\text { "Nursing" [DeCS] AND "Practice [DeCS] en el título; AND "Teaching" } \\
\text { [DeCS] AND "Advanced" [DeCS] en el resumen. }\end{array}$ & 4 \\
\hline $\begin{array}{l}\text { "Nursing" [DeCS] AND "Practice [DeCS] en el título; AND "Education" } \\
\text { [DeCS] AND "Advanced" [DeCS] en el resumen. }\end{array}$ & 27 \\
\hline $\begin{array}{l}\text { "Enfermería [DeCS] AND práctica" [DeCS] en el título; AND "Avances" } \\
\text { [DeCS] AND "Educación” [DeCS] en el resumen. }\end{array}$ & 1 \\
\hline $\begin{array}{l}\text { "Enfermería [DeCS] AND práctica" [DeCS] en el título; AND "Avances" } \\
\text { [DeCS] AND "Educación" [DeCS] en el resumen. }\end{array}$ & 0 \\
\hline
\end{tabular}

Fuente: elaboración propia

La búsqueda dio como resultado veintisiete artículos, de los cuales cuatro se repitieron en otros resultados. Es así como los veintitrés restantes fueron revisados en su totalidad.

4. Valoración de la calidad y la validez de las publicaciones. Los criterios incluidos en la ventana de observación garantizaban que fueran publicaciones indexadas. Luego de ubicarlas en texto completo, se hizo un análisis de ellas revisando en cada una qué tendencias conceptuales, teóricas o empíricas se reflejan en relación con el fortalecimiento de alianzas docencia-servicio para el avance de la enfermería.

5. Información obtenida y analizar los resultados. Se revisaron los resultados por agrupación temática, fechas, idioma y contenido.

\section{Resultados}

Las tendencias de publicación en relación con el fortalecimiento de alianzas docencia-servicio para el avance de la enfermería en los tres últimos años se pueden clasificar en tres grandes grupos, así:

1. Publicaciones con énfasis conceptual. El primer grupo lo conforman nueve publicaciones con énfasis conceptual, todas en idioma inglés, distribuidas en el tiempo así: tres publicaciones de 2013 (20-22), dos de 2014 $(23,24)$ y cuatro de 2015 (25-28).

Este grupo aborda temáticas referentes al empleo de marcos conceptuales para fortalecer las capacidades de la práctica clínica $(20,23)$, la importancia y el impacto que pueden tener las enfermeras formadas a nivel de doctorado en la práctica clínica (25), la pertinencia de los consensos para la práctica avanzada $(27,28)$, el tipo y ámbito de práctica que se puede tener $(21,22,24)$.

2. Publicaciones con énfasis teórico. El segundo grupo tiene énfasis teórico y está constituido por doce publicaciones, once de ellas en idioma inglés y una en español. Las fechas de publicación son una en 2013 (29), siete en 2014 (30-35) y cuatro en 2015 (36-39). 
3. Este grupo aborda temáticas referentes a las posibilidades de práctica avanzada en grupos específicos (30-36); el resultado de estar formado en un grupo interprofesional PS (36); la pertinencia de integrar las TIC (33) a la práctica avanzada; el ejercicio y la percepción de las prácticas avanzadas en campos clínicos específicos $(29,34,37,38)$ y los retos de la práctica avanzada $(35,39,40)$.

4. Publicaciones con énfasis empírico. El tercero y último de los grupos tiene un énfasis empírico y en este se encuentran dos publicaciones, ambas en idioma inglés, una de 2013 (41) y otra de 2014 (42).

Las temáticas que abordan son la comparación de técnicas de enseñanza basadas en la estandarización de pacientes para el fortalecimiento de las actividades comunicativas (41) y la descripción de la aplicación del modelo centrado en el paciente en el hogar (42).

\section{Discusión}

Al revisar las tendencias conceptuales, teóricas y empíricas en relación con el fortalecimiento de alianzas docencia-servicio para el avance de la enfermería se ve un claro predominio de los trabajos conceptuales o teóricos sobre los empíricos. Llama la atención que solo haya un estudio de tipo experimental, lo que evidencia el bajo desarrollo en términos de las posibilidades de emplear la teoría.

A pesar de que las organizaciones internacionales de enfermería han establecido programas para el desarrollo de la regulación que favorezca la práctica avanzada de la enfermería, existe a escala internacional una importante variación en los requisitos de formación, la regulación y su alcance que se ve restringido por normativas locales. A pesar de ello, la práctica de enfermería se sigue desarrollando en el ámbito mundial, debido a que la necesidad de cuidados de enfermería, el tener documentados los avances de la práctica y disponer de la información requerida en cada lugar pueden constituir una herramienta valiosa para su desarrollo y permitir un mayor impacto en las políticas relacionadas con la práctica avanzada de enfermería (26) y en el desempeño de las enfermeras que lideran el desarrollo de la práctica desde sus respectivos roles (35).

Se ha recomendado identificar y resolver aquellos temas que generan barreras para el desarrollo de la práctica clínica (35). En el caso colombiano, este ejercicio se ha desarrollado en el ámbito local y ha reflejado que el aumento de enfermeras que acceden a educación avanzada, las políticas de acreditación en salud, la facilidad de comunicación para acceder a recursos de investigación internacional y a redes de colaboración en investigación, así como la unión de esfuerzos de docencia-servicio que se ven en algunos ámbitos, generan un momento adecuado. Sin embargo, los autores señalan que es necesario trabajar por mayor autonomía en enfermería, mejorar incentivos para movilizar al talento humano hacia una mayor capacitación y desempeño del rol investigador, fomentar la práctica basada en la evidencia, así como mejorar la comunicación entre la academia y la asistencia (40). Se destaca el trabajo por competencias como un punto de encuentro entre la teoría y la práctica y entre la educación y la asistencia (23).

Es también evidente la presencia y el impacto de los niveles de formación más altos en la práctica, donde se espera que los profesionales con doctorado y maestría en enfermería hagan la diferencia mostrando impacto en términos de calidad en la práctica $(25,30,36)$, a pesar de que en algunos ámbitos de la práctica, aunque cada vez menos, se mantiene la controversia de preferir la experiencia sobre la educación y se rechaza la hegemonía de 
los doctores en la investigación (24). En América Latina, la necesidad de incrementar los niveles de maestría y superior en el ámbito clínico es un reto vigente (39).

Esta formación avanzada permitirá el descubrimiento de nuevos roles, como el de empresario, que contrastan con los hasta ahora descritos (3), y fortalecerá prácticas requeridas por las poblaciones más vulnerables como los niños maltratados (34), los enfermos crónicos, en especial aquellos que son atendidos por sus familias y requieren modelos innovadores de apoyo para sus situaciones específicas $(38,42)$.

También se sugiere el empleo de abordajes de modelos conceptuales de enfermería que permitan el manejo de diversas situaciones complejas que se presentan en la clínica, así como la clara definición de los roles que se desempeñan (26) y la delimitación del ámbito de las competencias en cada uno de ellos $(20,21)$ y la formación y la certificación que los respalde (22). Asimismo, se hace necesario tener presente que a medida que la práctica avanza, se hace más evidente la necesidad de profundizar en temas éticos como respaldo a decisiones autónomas (27).

Sobresale la importancia de retener en el rol asistencial a las enfermeras con alta preparación (31), quienes dan mayor coherencia e integralidad a la totalidad del sistema a favor del cuidado del paciente (38).

De otra parte, se sugiere evitar la hegemonía profesional casi total en la formación (24). Por ejemplo, la presencia de expertos en comunicación puede afectar la práctica de manera positiva (36). También se refleja la tendencia a valorar el empleo de TIC de punta para favorecer la práctica, para lo cual la formación debe ser adecuada (33).

La relación enfermera-paciente debe ser privilegiada. De una parte, puede y debe ser fortalecida desde la misma educación (41) y, de otra, el tener una relación adecuada genera implicaciones para que la práctica óptima de enfermería sea posible en diversos roles (32).

\section{Conclusiones}

El desarrollo de la práctica de enfermería centrada en el paciente se da a escala mundial, para lo cual la documentación y la socialización de avances resultan esenciales.

$\mathrm{Al}$ revisar las tendencias conceptuales, teóricas y empíricas en relación con el fortalecimiento de alianzas docencia-servicio para el avance de la enfermería se ve un claro predominio de los trabajos conceptuales o teóricos sobre los empíricos.

Identificar y resolver las barreras para el desarrollo de la práctica clínica es necesario para continuar con el desarrollo de esta política.

El trabajo por competencias es importante y necesario punto de encuentro entre la docencia, la asistencia y la investigación y a favor de una práctica avanzada.

En la práctica, la relación con el paciente y la mirada integral del sistema son esenciales. Se recomienda emplear mecanismos y tecnologías disponibles para que esta mirada y dicha relación se fortalezcan.

La formación más alta de enfermería en niveles de maestría y doctorado tiene un efecto positivo en la cualificación de la práctica, por lo cual es necesario promover oportunidades desde la asistencia y la docencia para permitir que esta formación se dé.

El avance y la complejidad de la práctica clínica traerá nuevos retos que la enfermería tendrá que asumir si pretende fortalecer su autonomía. 
Mantener y fortalecer la alianza docencia-servicio es una política vigente y necesaria que requiere estrategias concretas para la cualificación de la práctica de enfermería.

\section{Referencias bibliográficas}

1. Durán VM. Marco epistemológico de la enfermería. Aquichán. 2002;2(1):7-18.

2. Finkelmann A, Kenner C. Professional nursing concepts, tercera edición. Burlington, MA: Jones and Barlett; 2016.

3. Montealegre C. Roles de la enfermera clínica en un servicio de oncología. Revista Iberoamericana de Educación e Investigación en Enfermería [serie en internet]. 2014 [acceso: 11 de septiembre del 2015]; 1 [aprox 7 páginas]. Disponible en http://www. enfermeria21.com/revistas/aladefe/articulo/109/

4. Castro-Carvajal B. Los inicios de la profesionalización de la enfermería en Colombia. Investigación y Educación en Enfermería, 2011;29(2):269-85.

5. Letelier VM, Velandia MA. Profesionalismo en enfermería, el hábito de la excelencia del cuidado. Avances en Enfermería, 2010;28(2):145-58.

6. Gómez BE. La enfermería en Colombia: una mirada desde la sociología de las profesiones. Aquichán [serie en internet]. 2012 [acceso: 11 de septiembre del 2015];12(1):42-52. Disponible en: http://www. scielo.org.co/scielo.php?script $=$ sci arttext\&pi$\mathrm{d}=$ S1657-59972012000100005\&lng =en

7. Paravic-Klijn T. Enfermería y globalización. Cienc enferm. 2010;16(1):9-15.

8. Burgos MM, Paravic KT. Enfermería como profesión. Revista Cubana de Enfermería [serie en Internet]. 2009 Jun. [acceso: 12 de septiembre de 2015]; (25):1-2. Disponible en: http:// scielo.sld.cu/scielo.php?script $=$ sci_arttext $\&$ pi$\mathrm{d}=$ S0864-03192009000100010\&lng =es

9. Beltrán O. Revisiones sistemáticas de la literatura. Rev Colomb Gastroenterol. 2005;20(1):60-9.

10. Soto FP, García A. Impacto de un modelo de integración docente asistencial en la formación profesional y el campo clínico. Ciencia y Enfermería. 2011; 27(3):51-68.

11. Salgado PJ, Sanhueza AO. Enseñanza de la enfermería y relación docente asistencial en el marco educacional y sanitario chileno. Investigación y Educación en Enfermería [serie en internet]. 2010 [acceso: 13 de septiembre del 2015];28(2):258-66. Disponible en:http://www. scielo.org.co/scielo.php?script $=$ sci_arttext $\&$ pi$\mathrm{d}=$ S0120-53072010000200013\&lng=en\&tlng =es

12. Ministerio de Salud. El camino a seguir en el tema docencia servicio en salud [boletín de prensa n. ${ }^{\circ}$ 276 de 2013] [acceso 13 de septiembre del 2015]. Disponible en: https://www.minsalud.gov.co/Paginas/docencia-servicio-salud.aspx

13. Decreto 2376 de 2010, Presidencia de la República de Colombia, Por medio del cual se regula la relación docencia-servicio para los programas de formación de talento humano del área de la salud [acceso: 13 de septiembre del2015]. Disponible en: http://www.udea.edu.co/wps/wcm/connect/ udea/138d592c-92db-4394-bd5b-c8b7563a95e9/ Decreto_2376_de_Julio_01_de_2010.pdf?MO$\mathrm{D}=\mathrm{AJPERES}$

14. Resolución n. ${ }^{\circ}$ 3409, 23 de octubre del 2012, Ministerio de Salud y Protección Social de Colombia, Por la cual se define la documentación para efectos del reconocimiento de las Instituciones Prestadoras de Servicios de Salud como "Hospitales Universitarios" [acceso: 13 de septiembre del 2015]. Disponible en: http://www.alcaldiabogota.gov.co/ sisjur/normas/Norma1.jsp?i=50083

15. Asociación Colombiana de Facultades de Enfermería [sede web]. Plan de desarrollo Acofaen 2009-2018 [acceso: 13 de septiembre del 2015]; Disponible en: http://acofaen.org.co/quienes-somos/plan-de-desarrollo-2009-2018/

16. Carvallo SB. Entrevista telefónica realizada por Mónica Olarte a la presidenta de la Asociación Nacional de Enfermería de Colombia (ANEC), realizada el 23 de septiembre del 2015.

17. Carvajal HE. Entrevista personal realizada por Beatriz Sánchez a una representante del Grupo de Trabajo de Calidad de la Atención de Enfermería de siete IPS de Bogotá, realizada el 23 de septiembre del 2015.

18. Alarcón AM, Barrera OL, Carreño S, Carrillo GM, Farías RE, González G, Sánchez HB, Santamaría N. Desarrollo de un modelo funcional de cuidado de enfermería en cáncer. Investigación y Educación en Enfermería. 2014;32(2): 206-15.

19. Departamento Administrativo de Ciencia Tecnología e Innovación Colciencias. Convocatoria nacional para el reconocimiento y medición de grupos de investigación 2015. Desarrollo tecnológico o de innovación y para el reconocimiento de investigadores del SNCTeI-2014. Disponible en: http://www.colciencias.gov.co/articulos/medici-n-de-grupos-de-investigaci-n-desarrollo-tecnol-gico-yo-innovaci-n-y-recocimiento-de

20. Gardner G, Chang AM, Duffield C, Doubrovsky A. Delineating the practice profile of advanced practice nursing: a cross-sectional survey using the modified strong model of advanced practice. Journal of Advanced Nursing. 2013 Sep; 69(9):1931-42.

21. Lattimer JG. Population-based advanced practice nursing: where does oncology fit in? Clinical Journal of Oncology Nursing. 2013 Dec; 17 (6): 576-577.

22. Kleinpell RM, Hudspeth R. Issues in advanced practice. advanced practice nursing scope of practice for hospitals, acute care/critical care, and ambulatory care settings: a primer for clinicians, executives, and preceptors. Advanced Critical Care. 2013;24(1):23-9.

23. O'Connell J, Gardner G, Coyer F. Beyond competencies: using a capability framework in developing practice standards for advanced practice nursing. Journal of Advanced Nursing. 2014 Dec;70(2):2728-35. 
24. Dreher M, Clinton P, Sperhac A. Can the Institute of Medicine Trump the dominant logic of nursing? Leading change in advanced practice education. Journal of Professional Nursing. 2014 March-April; 30(2):104-9.

25. Paplham P, Austin KT. Doctor of nursing practice education: impact on advanced nursing practice. Seminars in Oncology Nursing. 2015 Nov; 31(4):273-81.

26. Heale, R, Rieck BC. An international perspective of advanced practice nursing regulation. International Nursing Review. 2015 Sep;62(3):421-9.

27. Laabs, CA. Toward a consensus in ethics education for the doctor of nursing practice. Nursing Education Perspectives. 2015Jul;36(4):249-51.

28. Kane CF. The 2014 scope and standards of practice for psychiatric mental health nursing: Key Updates. Online Journal of Issues in Nursing. 2015 Jan;20(1):1-4.

29. Swanson ML, Stanton M. Chief nursing officers' perceptions of the doctorate of nursing practice degree. Nursing Forum. 2013 Jan-Mar; 48(1):35-44.

30. Robazzi C, Do Carmo ML. Possibilities of advanced practices and professional master's programs in nursing. Revista Latino-Americana de Enfermagem. 2014 Sept/Oct;22(5):707-8.

31. East LA, Arudo J, Loefler M, Evans CM. Exploring the potential for advanced nursing practice role development in Kenya: a qualitative study. BMC Nursing [serie en internet]. 2014 Nov 14 [acceso: 12 de septiembre del 2015];13(33):1-11. Disponible en: http://www.biomedcentral.com/1472-6955/13/33

32. Dupin CM, Borglin G, Debout C, Rothan-Tondeur M. An ethnographic study of nurses' experience with nursing research and its integration in practice. Journal of Advanced Nursing. 2014 Sep;70(9):2128-39.

33. Swenty CL, Titzer JL. Faculty for faculty: a sense of urgency: integrating technology and informatics in advance practice nursing education in special issue: technology that transforms health care practice and education. The Journal for Nurse Practitioners. 2014 Nov-Dec;10(10):e57-e67.

34. Hornor G, Herendeen P. Advanced practice nursing in child maltreatment: practice characteristics. Journal of Pediatric Health Care. 2014 SepOct;28(5):438-43.
35. Kleinpell R, Scanlon A, Hibbert D, DeKeyser-Ganz F, East L, Fraser D, et al. Addressing issues impacting advanced nursing practice worldwide. Online Journal of Issues in Nursing [serie en internet] 2014 May [acceso: 13 de septiembre del 2015]; 19(2) [aprox. 5 páginas]. Disponible en: http://www.nursingworld.org/MainMenuCategories/ANAMarketplace/ANAPeriodicals/OJIN/ TableofContents/Vol-19-2014/No2-May-2014/ Advanced-Nursing-Practice-Worldwide.html

36. Defenbaugh N, Chikotas NE. The outcome of interprofessional education: Integrating communication studies into a standardized patient experience for advanced practice nursing students. Nurse Education in Practice [en prensa].

37. Walker DK, Polanich S. Doctor of nursing practice: the role of the advanced practice nurse. Seminars in Oncology Nursing. 2015;(31)4:263-73.

38. Underhill M, Roper K, Siefert M, Boucher J, Berry D. Evidence-based practice beliefs and implementation before and after an initiative to promote evidence-based nursing in an ambulatory oncology setting. Worldviews on Evidence-Based Nursing. 2015 Apr;12(2):70-78.

39. De Bortoli-Cassiani SH, Zug KE. The advanced practice nursing role in Latin America: challenges, opportunities and implications for universal health coverage. Investigación en Enfermería: Imagen y Desarrollo. 2015 ene-jun;17(1):9-11.

40. De Bruyn RR, Ochoa-Marín SC, Semenic S. Barreras y facilitadores en la práctica de la enfermería basada en la evidencia en Medellín, Colombia. Investigación y Educación en Enfermería. 2014 mar; 32(1):9-21.

41. Lin EC, Chen SL, Chao SY, Chen YC. Using standardized patient with immediate feedback and group discussion to teach interpersonal and communication skills to advanced practice nursing students. Nurse Education Today. 2013 Jun;33(6):677-83.

42. Swartwout K, Murphy MP, Dreher, MC, Behal R, Haines A, Ryan M, et al. Advanced practice nursing students in the patient-centered medical home: preparing for a new reality. Journal of Professional Nursing. 2014 Marz-Abr;30(2):139-48. 